\begin{tabular}{c} 
Volume and Issues Obtainable at Center for Sustainability Research and Consultancy \\
Journal of Accounting and Finance in Emerging Economies \\
ISSN: 2519-0318 ISSN (E) 2518-8488 \\
Volume 7: Issue 1 March 2021 \\
CSRC \\
Journal homepage: $\underline{\text { www.publishing.globalcsrc.org/jafee }}$ \\
\hline
\end{tabular}

\title{
Ease of Doing Business: Analysis of Trade Facilitations of One Hundred Twenty- Seven Countries of the World
}

\author{
${ }^{1}$ Tehmina Fiaz Qazi, ${ }^{2}$ Abdul Aziz Khan Niazi, ${ }^{3}$ Waheed Asghar, ${ }^{4}$ Abdul Basit \\ ${ }^{1}$ Hailey College of Banking and Finance, University of the Punjab, Lahore, Pakistan, \\ tehmina.qazi@gmail.com \\ ${ }^{2}$ Institute of Business and Management, University of Engineering and Technology, Lahore, Pakistan, \\ azizniazi@uet.edu.pk \\ ${ }^{3}$ Director in Technical Education and Vocational Training Authority, (Government of the Punjab), \\ Lahore, Pakistan,waheedasgharpk@gamil.com, director.at@tevta.gop.pk \\ ${ }^{4}$ Lahore Institute of Science \& Technology, Lahore, Pakistan, abasit_shahbaz@yahoo.com

\begin{tabular}{ll}
\hline \multicolumn{1}{c}{ ARTICLE DETAILS } & ABSTRACT \\
\hline $\begin{array}{l}\text { History } \\
\text { Revised format: February }\end{array}$ & $\begin{array}{l}\text { Aim of the study is to evaluate ease of doing business though } \\
\text { analysis of trade facilitations by different countries. The scope of the } \\
\text { study involves one hundred and twenty-seven countries of the world } \\
\text { available Online: } \text { March } \\
\text { and uses secondary data taken from World Development Indicators } \\
\text { (WDI) 2020. Overall design of study consists of review of literature, }\end{array}$ \\
\hline $\begin{array}{l}\text { Keywords } \\
\text { Business, countries, GRA, } \\
\text { Pakistan, trade facilitation, } \\
\text { ease of business. }\end{array}$ & $\begin{array}{l}\text { Analysis (GRA) as research methodology. Results show that } \\
\text { member countries of OECD performed exceptionally well, whereas, } \\
\text { member countries of SADC have exceptionally poor performance on }\end{array}$ \\
\hline JEL Classification: & agenda of ease of doing business and trade facilitations. The study is \\
M0, M1 & $\begin{array}{l}\text { useful for existing and potential business owners/mangers, policy } \\
\text { makers and researchers. It uses reliable country level original dataset } \\
\text { therefore findings of the study are valuable for stakeholders. }\end{array}$
\end{tabular}
OPEN $\bigcirc$ ACCESS (C) 2020 The authors, under a Creative Commons AttributionNonCommercial 4.0

Corresponding author's email address: azizniazi@uet.edu.pk

Recommended citation: Qazi, T. F., Niazi, A. A. K., Asghar, W. \& Basit, A. (2021). Ease of Doing Business: Analysis of Trade Facilitations of One Hundred Twenty-Seven Countries of the World. Journal of Accounting and Finance in Emerging Economies, 7(1), 65-75

\section{Introduction}

Inter-country trade can be relegated back to the history of hundreds of years. There are numerous explanations of international trade advanced by main stream economists by way of introducing international trade theories viz theory of absolute advantage, theory of comparative advantage and theory of mercantilism, etc. The literature is rich on theoretical explanations of international trade, however, the question of trade facilitation is a relative term investigated from time to time by wide range of researches in a wide variety of contexts. For example: Tang (2020) claimed that increase in level of trade facilitation has significant importance. Ismail (2020) proclaimed that having internet played significant role in trade facilitation; however it raised concerns about internet security and intensity of 
use. Martínez-Zarzoso and Rudolf (2020) found that co-ethic Chinese networks have positive impact on China's bilateral trade including machinery equipment, food sector, transport and raw material. Park and Park (2018) examined that financial investment on Authorized Economic Operator (AEO) certification has positive effect on performance through trade facilitation and public-private partnership. Sakyi et al. (2018) and Shepherd (2016) documented that better trade facilitation results in significant improvement in institutions, infrastructure and market efficiency. However, there is a dearth of a literature concerning comparison and/or ranking of the countries based on multiple variables in general and comprehensive study envisaged on data of whole world or at least envisaged on the countries whose data is conveniently and comprehensively available on websites of international institutions in particular. Trade facilitation is equally beneficial for businessmen and governments to particularly eliminate corruption. It is also beneficial for businesses and countries by way of accessing markets (both international supplier markets and consumer markets). It is also beneficial to support and participate in global value chains. It is call of the day to assess the country level position in terms of trade facilitation qua rivals. Therefore, the problem under investigation here is, assessing the ease of doing business, particularly international trade and country level analysis of trade facilitation which has not been addressed in contemporary literature as such. Objectives of the study include ranking 127 countries on the basis of Grey Relational Grades (GRGs) based on seven different criteria. Further, to classify the countries on the continuum of ordinal scale divided into seven items viz exceptionally good, very good, good, fair, poor, very poor and exceptionally poor. For achieving these objective wide varieties of methodologies was considered e.g. ANP, FANP, DEMATEL, AHP, PROMETHEE, TOPSIS, DEA, GRA, VIKOR, SWARA, ISM, TISM, MICMAC, FMICMAC, IPA, ELECTRE, NSGA-II, MADM, MAGDM, ARAS-F, FMEA-Model, ELECTRE-III, COPRAS-G, MULTI-MOORA, ARAS, WASPAS, MOORA, LP, IRP, SEM and Regression. GRA was found to be the most appropriate methodology; therefore, this study uses GRA. Rest of the paper is arranged as literature review, methodology, results \& discussions and conclusion.

\section{Literature Review}

We have searched the literature in research data bases like Emerald, Elsevier (ScienceDirect), WileyBlackwell, Springer and Taylor \& Francis, etc. using key words 'trade facilitations', 'ease of business', 'international trade', etc. and found plethora of research. Some highly relevant studies are reported here to set the outset of study e.g. the studies on trade facilitation policies of Shenzhen and Hong Kong (Cheng et al. 2018), role of trade facilitation in Central Asia-Azerbaijan and Tajikistan (Felipe \& Kumar, 2012), trade facilitation agreement policies in French firms (Fontagné et al., 2020), impact of trade facilitation on trade of developing countries (Indicators, 2013), impact of trade facilitation of South Africa's exports (Jordaan, 2014), cross-border trade and trade facilitation in Sub-Saharan Africa (Lesser \& Moisé-Leeman, 2009), prominent role of trade facilitation in Middle-East (Marti et al., 2014), impact of trade facilitation on tourism in Japan (Tang, 2020), Chinese diaspora and trade facilitation in Australia (Tung \& Chung, 2010), examine the trade policy of India (Ural Marchand, 2019), tradefacilitated technology across EU countries (Wan et al., 2015), trade facilitation and corruption Egyptian model (Zaki, 2013). Feenstra and Ma (2014) determined positive significant impact of trade between OECD (Organization for Economic Cooperation and Development) \& non-OECD member countries and countries without common borders. Gnangnon (2017) analyzed that trade facilitation reforms that have positive impact on tax revenue. Hoekman and Nicita (2010) asserted that less attention on facilitation of trade has adverse impact on trade expansion initiative. Martinez-Zarzoso and MárquezRamos (2008) affirmed that initiatives taken in better trade facilitations policies have significant positive impact on increasing trade and building trust among trading partners. Shepherd (2016) asserted that APEC (Asia Pacific Economic Cooperation) has taken constructive initiatives to reduce trading costs, border procedures and customs to facilitate trade across borders. Valensisi et al. (2016) argued that there is negative effect of trade-related costs on trade facilitation and positive significant effect of trade free area on trade facilitation. Dennis \& Shepherd (2011); Iwanow \& Kirkpatrick (2007) and Wilson et al. (2005) bolstered that trade facilitation reforms is likely to be associated with export expansion. Lot of research, in fact, has surpassed on trade facilitation and export expansion that is reviewed but not being reported here to maintain brevity of the study. 


\section{Theoretical Framework}

Theoretical framework of the study fixes limits of study and underlines approach and methodology including dataset \& probable solution to the research problem. In general this study relies on the theoretical foundations of WDI (2020). The variable specification and definitions have also been adopted from WDI (2020). Variables of the study thus have been specified as follows.

Table 1: Description of Variables

\begin{tabular}{clll}
\hline Code & \multicolumn{1}{c}{ Variable of Trade Facilitation } & Scale of Measurement & \multicolumn{1}{c}{ Criteria } \\
\hline $\mathbf{1}$ & Logistics performance index & $1-5$ (worst to best) & Larger is the best \\
$\mathbf{2}$ & Burden of customs procedures & $1-7$ (worst to best) & Larger is the best \\
$\mathbf{3}$ & Lead Time to Export & No. of Days & Smaller is the best \\
$\mathbf{4}$ & Lead Time to Imports & No. of Days & Smaller is the best \\
$\mathbf{5}$ & Documentary Compliance Cost to Export & US Dollars & Smaller is the best \\
$\mathbf{6}$ & Documentary Compliance Cost to Import & US Dollars & Smaller is the best \\
$\mathbf{7}$ & Quality of port infrastructure & $1-7$ (worst to best) & Larger is the best \\
\hline
\end{tabular}

For further strengthening the theoretical foundations, findings of few studies are reported here. Cheng et al. (2018) and Feenstra and Ma (2014) concluded that development of efficient port infrastructure has potential impact on increase in exports. Jordaan (2014); Messerlin \& Zarrouk (2000) and Seck (2016) reported that improvement in customs procedures and government regulations lead to gain better importing policy that results in increase in trade flows. Manzoor et al. (2019) identified some major challenges in embarking on regime of trade facilitation e.g. poor infrastructure, prolonged customs procedure, poor trade logistics, high import duties and high tariffs in regional trade. Marti et al. (2014) highlighted the importance of trade facilitation in Middle-East exporters and revealed that logistics index performs well in complex goods transportation. Felipe and Kumar (2012) stated that development of infrastructure; improve logistics and efficient customs policies have great importance for trade facilitation. Lorz (2020) bolstered that cooperation between different trading countries in development of infrastructure can decrease cost of logistics, cost to import, cost to export and facilitate trading. Dennis (2010) and Nguyen Viet (2015) argued that lead time to imports \& exports and complexity of documentation are major determinants of levels of trade facilitation. Fontagné et al. (2020) buttressed that automation and simplification of documents have positive impact on large firms' trade.

\section{Methodology}

It is a quantitative research study following positivism as research philosophy that uses statistical secondary data of population of 127 countries for deducing inference on country level trade facilitation. Methodology entails on literature review, extraction of data and mathematical analysis. The methodology of the study is espoused in grey system theory. Grey system theory consists of five parts: grey prediction, grey relational analysis, grey decision, grey programming and grey control (Ju-Long, 1989; Wei, 2011). We used Grey Relational Analysis (GRA) for evaluating the trade facilitations. Classical procedure of GRA (Hamzaçebi \& Pekkaya, 2011; Kuo et al., 2008; Tayyar et al., 2014; Wu, 2002) is applied. GRA has also been amplified with classification of countries (Qazi et al., 2020). The process and symbols used in the study have been adopted from Ertuğrul et al. (2016).

Step 1: Created a dataset and established decision matrix Eq. (1)

$$
x_{i}(k)=\left[\begin{array}{ccc}
x_{1}(1) x_{1}(2) & \cdots & x_{1}(m) \\
\vdots & \ddots & \vdots \\
x_{n}(1) x_{n}(2) & \cdots & x_{n}(m)
\end{array}\right] \quad \text { Eq. (1) }
$$

Table 2: Trade Facilitation Data of Countries

\begin{tabular}{ccccccccc}
\hline Sr. & Country & $\mathbf{1}$ & $\mathbf{2}$ & $\mathbf{3}$ & $\mathbf{4}$ & $\mathbf{5}$ & $\mathbf{6}$ & $\mathbf{7}$ \\
\hline $\mathbf{1}$ & Albania & 2.66 & 4 & 10 & 14 & 10 & 10 & 4.1 \\
\hline
\end{tabular}




\begin{tabular}{clccccccc}
\hline $\mathbf{2}$ & Algeria & 2.45 & 3.4 & 4 & 5 & 374.4 & 399.8 & 3.4 \\
$\ldots$ & $\ldots \ldots \ldots \ldots$ & $\ldots$ & $\ldots$ & $\ldots$ & $\ldots$ & $\ldots$ & $\ldots$ & $\ldots$ \\
$\ldots$ & $\ldots \ldots \ldots \ldots$ & $\ldots$ & $\ldots$ & $\ldots$ & $\ldots$ & $\ldots$ & $\ldots$ & $\ldots$ \\
$\mathbf{9 1}$ & Pakistan & 2.42 & 3.7 & 4 & 8 & 118 & 130 & 4 \\
$\mathbf{9 2}$ & Panama & 3.28 & 4.5 & 3 & 2 & 60 & 50 & 6.2 \\
$\ldots$ & $\ldots \ldots \ldots \ldots$ & $\ldots$ & $\ldots$ & $\ldots$ & $\ldots$ & $\ldots$ & $\ldots$ & $\ldots$ \\
$\ldots$ & $\ldots \ldots \ldots \ldots$ & $\ldots$ & $\ldots$ & $\ldots$ & $\ldots$ & $\ldots$ & $\ldots$ & $\ldots$ \\
$\mathbf{1 2 6}$ & Zambia & 2.53 & 3.7 & 9 & 6 & 200 & 175 & 2.3 \\
$\mathbf{1 2 7}$ & Zimbabwe & 2.12 & 2.9 & 5 & 10 & 170 & 150 & 3.1 \\
\hline \multicolumn{6}{l}{ Source of Data: $[$ World Development Indicators (WDI), 2020] }
\end{tabular}

Step 2: Created reference series and comparison matrix Eq. (2)

$$
x_{0}=\left[x_{0}(k) \ldots \ldots \ldots \ldots x_{0}(n)\right] \quad E q .(2)
$$

Table 3: Reference Sequence and Comparable Sequences

\begin{tabular}{|c|c|c|c|c|c|c|c|c|}
\hline Sr. & Country & 1 & 2 & 3 & 4 & 5 & 6 & 7 \\
\hline $\mathbf{0}$ & Reference Sequence & 4.2 & 6.3 & 1 & 1 & 0 & 0 & 6.8 \\
\hline $\mathbf{1}$ & Albania & 2.66 & 4 & 10 & 14 & 10 & 10 & 4.1 \\
\hline 2 & Algeria & 2.45 & 3.4 & 4 & 5 & 374.4 & 399.8 & 3.4 \\
\hline$\cdots$ & …....... & $\cdots$ & .. & $\cdots$ & $\cdots$ & $\cdots$ & .. & ... \\
\hline$\cdots$ & ........... & ... & $\ldots$ & ... & $\cdots$ & ... & ... & ... \\
\hline 91 & Pakistan & 2.42 & 3.7 & 4 & 8 & 118 & 130 & 4 \\
\hline 92 & Panama & 3.28 & 4.5 & 3 & 2 & 60 & 50 & 6.2 \\
\hline$\cdots$ & .. & .. & .. & $\cdots$ & $\cdots$ & .. & $\cdots$ & ... \\
\hline$\ddot{1}$ & $\cdots$ & $\cdots$ & .. & $\cdots$ & $\cdots$ & $\cdots$ & $\cdots$ & $\cdots$ \\
\hline 126 & Zambia & 2.53 & 3.7 & 9 & 6 & 200 & 175 & 2.3 \\
\hline 127 & Zimbabwe & 2.12 & 2.9 & 5 & 10 & 170 & 150 & 3.1 \\
\hline
\end{tabular}

Step 3: Created a normalized matrix for larger and smaller acceptable as the case may be Eq. (3) and Eq. (4)

1- For larger the better

$$
x_{i}^{*}(k)=\frac{x_{i}^{(0)}(k)-\min x_{i}^{(0)}(k)}{\max x_{i}^{(o)}(k)-\min x_{i}^{(o)}(k)} \quad E q .(3)
$$

2- For smaller the better

$$
x_{i}(k)=\frac{\max x_{i}^{(o)}(k)-x_{i}^{(0)}(k)}{\max x_{i}^{(0)}(k)-\min x_{i}^{(0)}(k)} \quad \text { Eq. }(4)
$$

\begin{tabular}{|c|c|c|c|c|c|c|c|c|}
\hline Sr. & Country & 1 & 2 & 3 & 4 & 5 & 6 & 7 \\
\hline $\mathbf{0}$ & Reference Sequence & 1.0000 & 1.0000 & 1.0000 & 1.0000 & 1.0000 & 1.0000 & 1.0000 \\
\hline 1 & Albania & 0.2837 & 0.4889 & 0.5714 & 0.5806 & 0.9800 & 0.9902 & 0.5000 \\
\hline 2 & Algeria & 0.1860 & 0.3556 & 0.8571 & 0.8710 & 0.2512 & 0.6100 & 0.3704 \\
\hline$\cdots$ & ........... & ... & ... & ... & ... & ... & ... & $\cdots$ \\
\hline$\dddot{91}$ & …...... & $\dddot{0}$ & ‥ & ‥ & ㄱ. & $\ldots$ & ‥ & $\ldots$ \\
\hline 92 & Panama & 0.5721 & 0.6000 & 0.9048 & 0.9677 & 0.8800 & 0.9512 & 0.8889 \\
\hline$\ldots$ & ........... & ... & ... & ... & ... & ... & ... & ... \\
\hline$\ldots$ & n......... & $\ldots$ & $\cdots$ & .. & .. & $\cdots$ & $\cdots$ & $\cdots$ \\
\hline 126 & Zambia & 0.223256 & 0.4222 & 0.6190 & 0.8387 & 0.6000 & 0.8293 & 0.1667 \\
\hline 127 & Zimbabwe & 0.032558 & 0.2444 & 0.8095 & 0.7097 & 0.6600 & 0.8537 & 0.3148 \\
\hline
\end{tabular}

Table 4: Normalized Comparable Sequences

For example, calculation of Albania, "logistics performance index" (1), larger is the best.

$$
x_{1}^{*}(1)=\frac{x_{1}^{0}(1)-\min x_{1}^{0}(1)}{\max x_{1}^{0}(1)-\min x_{1}^{o}(1)}=\frac{2.66-2.05}{4.20-2.05}=0.2837
$$


Step 4: Obtained absolute values by calculating deviation sequence Eq. (5)

$$
\Delta_{0 i}(k)=\left|x_{0}^{*}(k)-x_{i}^{*}(k)\right| \quad E q .(5)
$$

For biggest deviation Eq. (6)

$$
\Delta_{\text {max }}=\max _{\forall j E i} \max _{\forall k}\left|x_{0}^{*}(k)-x_{j}^{*}(k)\right| \quad E q .(6)
$$

For smallest deviation Eq. (7)

$$
\Delta_{\min }=\min _{\forall j \varepsilon i} \min _{\forall k}\left|x_{0}^{*}(k)-x_{j}^{*}(k)\right| \quad E q .(7)
$$

\begin{tabular}{|c|c|c|c|c|c|c|c|c|}
\hline Sr. & Country & 1 & 2 & 3 & 4 & 5 & 6 & 7 \\
\hline $\mathbf{0}$ & Reference Sequence & 0.0000 & 0.0000 & 0.0000 & 0.0000 & 0.0000 & 0.0000 & 0.0000 \\
\hline 1 & Albania & 0.7163 & 0.5111 & 0.4286 & 0.4194 & 0.0200 & 0.0098 & 0.5000 \\
\hline 2 & Algeria & 0.8140 & 0.6444 & 0.1429 & 0.1290 & 0.7488 & 0.3900 & 0.6296 \\
\hline$\ldots$ & ........... & ... & ... & ... & ... & ... & ... & ... \\
\hline$\ldots$ & ........... & ... & ... & ... & ... & ... & ... & ... \\
\hline 91 & Pakistan & 0.8279 & 0.5778 & 0.1429 & 0.2258 & 0.2360 & 0.1268 & 0.5185 \\
\hline 92 & Panama & 0.4279 & 0.4000 & 0.0952 & 0.0323 & 0.1200 & 0.0488 & 0.1111 \\
\hline$\ldots$ & ........... & ... & ... & ... & ... & ... & ... & ... \\
\hline$\ldots$ & ........... & ... & ... & ... & ... & ... & ... & ... \\
\hline 126 & Zambia & 0.7767 & 0.5778 & 0.3810 & 0.1613 & 0.4000 & 0.1707 & 0.8333 \\
\hline 127 & Zimbabwe & 0.9674 & 0.7556 & 0.1905 & 0.2903 & 0.3400 & 0.1463 & 0.6852 \\
\hline
\end{tabular}

Table 5: Deviation Sequences

For example, calculation of deviation of Algeria for "burden of customs procedures" (2)

$$
\Delta_{02}(2)=\left|x_{0}^{*}(2)-x_{2}^{*}(2)\right|=|1-0.3556|=0.6444
$$

Step 5: Grey relational co-efficient is calculated $E q$. (8). Term $\xi$ is distinguishing co-efficient between 0

\begin{tabular}{|c|c|c|c|c|c|c|c|c|}
\hline Sr. & Country & 1 & 2 & 3 & 4 & 5 & 6 & 7 \\
\hline $\mathbf{0}$ & Reference Sequence & 1.0000 & 1.0000 & 1.0000 & 1.0000 & 1.0000 & 1.0000 & 1.0000 \\
\hline 1 & Albania & 0.4111 & 0.4945 & 0.5385 & 0.5439 & 0.9615 & 0.9809 & 0.5000 \\
\hline 2 & Algeria & 0.3805 & 0.4369 & 0.7778 & 0.7949 & 0.4004 & 0.5618 & 0.4426 \\
\hline$\cdots$ & ........... & .. & ... & ... & $\cdots$ & ... & ... & $\cdots$ \\
\hline$\cdots$ & .......... & $\ldots$ & $\cdots$ & $\ldots$ & $\cdots$ & ... & $\cdots$ & $\cdots$ \\
\hline 91 & Pakistan & 0.3765 & 0.4639 & 0.7778 & 0.6889 & 0.6793 & 0.7977 & 0.4909 \\
\hline 92 & Panama & 0.5388 & 0.5556 & 0.8400 & 0.9394 & 0.8065 & 0.9111 & 0.8182 \\
\hline$\cdots$ & ........... & $\cdots$ & $\cdots$ & $\cdots$ & $\cdots$ & $\cdots$ & $\cdots$ & $\cdots$ \\
\hline$\cdots$ & $\cdots$ & $\cdots$ & $\cdots$ & $\cdots$ & .. & .. & $\cdots$ & $\cdots$ \\
\hline 126 & Zambia & 0.3916 & 0.4639 & 0.5676 & 0.7561 & 0.5556 & 0.7455 & 0.3750 \\
\hline 127 & Zimbabwe & 0.3407 & 0.3982 & 0.7241 & 0.6327 & 0.5952 & 0.7736 & 0.4219 \\
\hline
\end{tabular}
and 1 the usual value of which is 0.5 in literature.

$$
\gamma\left[x_{0}^{*}(k), x_{i}^{*}(k)\right]=\frac{\Delta_{\min }+\xi \Delta_{\max }}{x_{0 i}(k)+\xi \Delta_{\max }}, 0<\gamma\left[x_{o}^{*}(k), x_{i}^{*}(k)\right] \leq 1
$$

Table 6: Grey Relational Co-efficient

For example, calculation of grey relational of Algeria co-efficient for "burden of customs procedures" (2)

$$
\gamma\left[x_{0}^{*}(2), x_{2}^{*}(2)\right]=\frac{\Delta_{\min +\xi} \Delta_{\max }}{\Delta_{2}(2)+\xi \Delta_{\max }}=\frac{0+(0.5) \times 1}{0.6444+(0.5) \times 1}=0.4369
$$

Step 6: Weighted sum of grey relational co-efficient (Grey Relational Grade) Eq. (9) and Eq. (10).

$$
\gamma\left(x_{0}^{*}, x_{i}^{*}\right)=\sum_{k=1}^{n} \beta_{k} \gamma\left[x_{0}^{*}(k), x_{i}^{*}(k)\right] \quad E q .(9)
$$




$$
\sum_{k=1}^{n} \beta_{k}=1 \quad \text { Eq. (10) }
$$

Table 7: Grey Relational Grade (GRG)

\begin{tabular}{|c|c|c|}
\hline Sr. & Country & GRG \\
\hline 0 & Reference Sequence & 1.0000 \\
\hline 1 & Albania & 0.6329 \\
\hline 2 & Algeria & 0.5421 \\
\hline$\ldots$ & ........... & $\ldots$ \\
\hline$\ddot{01}$ & ........... & 06107 \\
\hline $\begin{array}{l}91 \\
92\end{array}$ & $\begin{array}{l}\text { Pakistan } \\
\text { Panama }\end{array}$ & 0.7728 \\
\hline$\cdots$ & ............ & ... \\
\hline ... & ........... & $\ldots$ \\
\hline 126 & Zambia & 0.5507 \\
\hline 127 & Zimbabwe & 0.5552 \\
\hline
\end{tabular}

For example, calculation of GRG for Algeria:

$$
\begin{gathered}
\gamma\left(x_{0}^{*}, x_{2}^{*}\right)=\sum_{k=1}^{n} \beta_{k} \gamma\left[x_{0}^{*}(2), x_{2}^{*}(k)\right] \\
=0.1429 \times(0.3805+0.4369+0.7778+0.7949+0.4004+0.5618+0.4426)=0.5421
\end{gathered}
$$

GRA has been amplified with a classification (Niazi et al., 2020) in order to assemblage the results of GRA (i.e. GRG) on a continuum of scale of seven items viz exceptionally good, very good, good, fair, poor, very poor and exceptionally poor. This augmentation of methodology facilitates the interpretations

\begin{tabular}{|c|c|c|}
\hline Sr. & Scale & Description \\
\hline 1 & $\begin{array}{l}\text { Exceptionally } \\
\text { Good }\end{array}$ & $\begin{array}{l}\text { Countries having GRG between } 0.9411-0.8141 \text { are considered having exceptionally good level } \\
\text { of trade facilitations. }\end{array}$ \\
\hline 2 & Very Good & $\begin{array}{l}\text { Countries having GRG between } 0.8097-0.7555 \text { are considered having very good level of trade } \\
\text { facilitations. }\end{array}$ \\
\hline 3 & Good & $\begin{array}{l}\text { Countries having GRG between } 0.7508-0.7112 \text { are considered having good level of trade } \\
\text { facilitations. }\end{array}$ \\
\hline 4 & Fair & $\begin{array}{l}\text { Countries having GRG between } 0.7106-0.6634 \text { are considered having level of fair level of trade } \\
\text { facilitations. }\end{array}$ \\
\hline 5 & Poor & $\begin{array}{l}\text { Countries having GRG between } 0.6620-0.6368 \text { are considered having poor level of trade } \\
\text { facilitations. }\end{array}$ \\
\hline 6 & Very Poor & $\begin{array}{l}\text { Countries having GRG between } 0.5934-0.5934 \text { are considered having very poor level of trade } \\
\text { facilitations. }\end{array}$ \\
\hline 7 & $\begin{array}{l}\text { Exceptionally } \\
\text { Poor }\end{array}$ & $\begin{array}{l}\text { Countries having GRG between } 0.5794-0.4166 \text { are considered having exceptionally poor level of } \\
\text { trade facilitations. }\end{array}$ \\
\hline
\end{tabular}
and adds value to the understanding of readers.

Table 8: Scheme of Classification

Readers will find classification significantly helpful in making an informed opinion about trade facilitations by different countries.

\section{Results \& Discussions}

\subsection{Results}

Inter-country trade and its facilitation have fundamental importance for economic growth and harmonization of political relationship. This study has addressed the problem of putting the countries into an order on the basis of their performance on trade facilitation. It is a study of 127 countries accounting for seven different criteria as proxy to trade facilitation. Aim of the study is to evaluate ease of doing business though analysis of trade facilitations by different countries. Grey relational analysis has been conducted to rank the countries result of which is given as Table 9 . 
Table 9: Results of GRA of Trade Facilitation

\begin{tabular}{|c|c|c|c|c|c|c|c|c|}
\hline Country & GRGs & Rank & Country & GRGs & Rank & Country & GRGs & Rank \\
\hline Reference Sequence & 1.0000 & 0 & Georgia & 0.7395 & 42 & Kyrgyz Republic & 0.6391 & 85 \\
\hline \multicolumn{2}{|c|}{ Exceptionally Good } & & Czech Republic & 0.7388 & 43 & Montenegro & 0.6379 & 86 \\
\hline Netherlands & 0.9411 & 1 & Bulgaria & 0.7348 & 44 & Cambodia & 0.6379 & 87 \\
\hline Singapore & 0.9226 & 2 & North Macedonia & 0.7347 & 45 & Qatar & 0.6376 & 88 \\
\hline Finland & 0.8888 & 3 & South Africa & 0.7257 & 46 & Guatemala & 0.6376 & 89 \\
\hline $\begin{array}{l}\text { Hong Kong SAR, } \\
\text { China }\end{array}$ & 0.8871 & 4 & Brunei Darussalam & 0.7203 & 47 & Mozambique & 0.6368 & 90 \\
\hline Belgium & 0.8764 & 5 & Dominican Republic & 0.7163 & 48 & \multicolumn{3}{|c|}{ Very Poor } \\
\hline Germany & 0.8667 & 6 & Ecuador & 0.7157 & 49 & Lao PDR & 0.6358 & 91 \\
\hline Sweden & 0.8661 & 7 & Serbia & 0.7152 & 50 & Albania & 0.6329 & 92 \\
\hline Norway & 0.8527 & 8 & $\begin{array}{l}\text { Bosnia and } \\
\text { Herzegovina }\end{array}$ & 0.7124 & 51 & Mongolia & 0.6317 & 93 \\
\hline United Kingdom & 0.8510 & 9 & China & 0.7122 & 52 & Iran, Islamic Rep. & 0.6304 & 94 \\
\hline Denmark & 0.8492 & 10 & Oman & 0.7118 & 53 & Burkina Faso & 0.6304 & 95 \\
\hline Austria & 0.8338 & 11 & Turkey & 0.7112 & 54 & Sri Lanka & 0.6283 & 96 \\
\hline Estonia & 0.8328 & 12 & \multicolumn{2}{|c|}{ Fair } & & Uruguay & 0.6263 & 97 \\
\hline Spain & 0.8289 & 13 & Canada & 0.7106 & 55 & Senegal & 0.6261 & 98 \\
\hline Luxembourg & 0.8278 & 14 & Morocco & 0.7073 & 56 & Ukraine & 0.6211 & 99 \\
\hline Iceland & 0.8245 & 15 & Philippines & 0.7024 & 57 & Kazakhstan & 0.6183 & 100 \\
\hline United States & 0.8235 & 16 & Gambia, The & 0.7017 & 58 & Kuwait & 0.6147 & 101 \\
\hline France & 0.8231 & 17 & India & 0.6975 & 59 & Pakistan & 0.6107 & 102 \\
\hline Poland & 0.8141 & 18 & Rwanda & 0.6957 & 60 & Egypt, Arab Rep. & 0.6065 & 103 \\
\hline \multicolumn{3}{|c|}{ Very Good } & Mauritius & 0.6940 & 61 & Libya & 0.5982 & 104 \\
\hline Switzerland & 0.8097 & 19 & Peru & 0.6893 & 62 & Myanmar & 0.5980 & 105 \\
\hline Slovenia & 0.8095 & 20 & Thailand & 0.6865 & 63 & Brazil & 0.5956 & 106 \\
\hline Portugal & 0.8090 & 21 & Lebanon & 0.6853 & 64 & Nicaragua & 0.5936 & 107 \\
\hline United Arab Emirates & 0.8079 & 22 & Jamaica & 0.6833 & 65 & Tunisia & 0.5934 & 108 \\
\hline Malta & 0.8006 & 23 & Colombia & 0.6803 & 66 & Exception & ly Poor & \\
\hline Japan & 0.7984 & 24 & Madagascar & 0.6724 & 67 & Tanzania & 0.5794 & 109 \\
\hline Korea, Rep. & 0.7928 & 25 & Mexico & 0.6672 & 68 & Uganda & 0.5766 & 110 \\
\hline Hungary & 0.7897 & 26 & Nepal & 0.6666 & 69 & Benin & 0.5734 & 111 \\
\hline Malaysia & 0.7798 & 27 & Indonesia & 0.6664 & 70 & Bangladesh & 0.5701 & 112 \\
\hline Latvia & 0.7795 & 28 & Costa Rica & 0.6638 & 71 & Nigeria & 0.5640 & 113 \\
\hline Italy & 0.7767 & 29 & Vietnam & 0.6634 & 72 & Liberia & 0.5573 & 114 \\
\hline Ireland & 0.7759 & 30 & \multicolumn{2}{|l|}{ Poor } & & Zimbabwe & 0.5552 & 115 \\
\hline Panama & 0.7728 & 31 & Jordan & 0.6620 & 73 & Zambia & 0.5507 & 116 \\
\hline Cyprus & 0.7694 & 32 & Bolivia & 601 & 74 & $\begin{array}{l}\text { Trinidad and } \\
\text { Tobago }\end{array}$ & 0.5440 & 117 \\
\hline Lithuania & 0.7675 & 33 & Haiti & 0.6527 & 75 & Algeria & 0.5421 & 118 \\
\hline Croatia & 0.7659 & 34 & Russian Federation & 0.6526 & 76 & Malawi & 0.5307 & 119 \\
\hline Romania & 0.7598 & 35 & Namibia & 0.6510 & 77 & Cameroon & 0.5096 & 120 \\
\hline Israel & 0.7555 & 36 & Cote d'Ivoire & 0.6492 & 78 & Tajikistan & 0.4755 & 121 \\
\hline \multicolumn{2}{|c|}{ Good } & & Argentina & 0.6424 & 79 & Congo, Dem. Rep. & 0.4633 & 122 \\
\hline Bahrain & 0.7508 & 37 & Honduras & 0.6423 & 80 & Mauritania & 0.4593 & 123 \\
\hline Australia & 0.7498 & 38 & Azerbaijan & 0.6406 & 81 & Angola & 0.4417 & 124 \\
\hline Slovak Republic & 0.7498 & 39 & Kenya & 0.6402 & 82 & Venezuela, RB & 0.4399 & 125 \\
\hline Chile & 0.7487 & 40 & Saudi Arabia & 0.6402 & 83 & Burundi & 0.4331 & 126 \\
\hline Greece & 0.7403 & 41 & Ghana & 0.6394 & 84 & Chad & 0.4166 & 127 \\
\hline
\end{tabular}

Result of GRA reveals that Netherlands, Singapore, Finland, Hong Kong SAR, China, Belgium, Germany, Sweden, Norway, United Kingdom, Denmark, Austria, Estonia, Spain, Luxembourg, Iceland, United States, France and Poland have exceptionally good level trade facilitations. Most of the countries under this ensign are member countries of Organization for Economic Co-operation and Development (OECD). Switzerland, Slovenia, Portugal, United Arab Emirates, Malta, Japan, Korea, Rep. Hungary, Malaysia, Latvia, Italy, Ireland, Panama, Cyprus, Lithuania.

Croatia, Romania and Israel are very good. Most of which are again member countries of OECD. 
Bahrain, Australia, Slovak Republic, Chile, Greece, Georgia, Czech Republic, Bulgaria, North Macedonia, South Africa, Brunei Darussalam, Dominican Republic, Ecuador, Serbia, Bosnia and Herzegovina, China, Oman and Turkey are good. Canada, Morocco, Philippines, Gambia, India, Rwanda, Mauritius, Peru, Thailand, Lebanon, Jamaica, Colombia, Madagascar, Mexico, Nepal, Indonesia, Costa Rica and Vietnam are fair, most of which are member countries of Asia Pacific Economic Cooperation (APEC). Jordan, Bolivia, Haiti, Russian Federation, Namibia, Cote d'Ivoire, Argentina, Honduras, Azerbaijan, Kenya, Saudi Arabia, Ghana Kazakhstan, Kuwait, Pakistan, Egypt, Arab Rep., Libya, Myanmar, Brazil, Nicaragua, and Tunisia poor in trade facilitation most of which are member countries of Arabian Countries (AC).. Tanzania, Uganda, Benin, Bangladesh, Nigeria, Liberia, Zimbabwe, Zambia, Trinidad and Tobago, Algeria, Malawi, Cameroon, Tajikistan, Congo, Dem. Rep., Mauritania, Angola, Venezuela, RB Burundi and Chad have exceptionally poor trade facilitation according to GRA. Most of these countries are member countries Southern Africa Development Community (SADC).

\section{Discussion}

Objectives of the study include ranking 127 countries on the basis of Grey Relational Grades (GRGs) based on seven different criteria. This objective has been achieved through GRA and GRA per se is different a type of methodology from contemporary methodologies. Scope of the study is also different and wider than previous literature. Number of variables accommodated within the methodology is also a distinctive feature of the current study. However, the results are synchronized and espoused in the existing literature. A contrast of current study with some of highly relevant studies from literature is represented below to enrich the understanding Table 10.

Table 10: Comparison with Contemporary Studies

\begin{tabular}{|c|c|c|c|c|}
\hline Studies & Focus & Variables/Factors & Methodology & Results \\
\hline Current & & $\begin{array}{l}\text { Logistics performance index, quality } \\
\text { of port infrastructure, burden of } \\
\text { customs procedures, lead time to } \\
\text { export \& imports, documentary } \\
\text { compliance cost to export \& import }\end{array}$ & $\begin{array}{l}\text { Grey relational } \\
\text { analysis }\end{array}$ & \\
\hline $\begin{array}{l}\text { Fontagné } \\
\text { et al. } \\
\text { (2020) }\end{array}$ & $\begin{array}{l}\text { Hetergenous } \\
\text { effects of trade } \\
\text { facilitation } \\
\text { measures }\end{array}$ & $\begin{array}{l}\text { time to export into a given market, } \\
\text { number of documents needed to be } \\
\text { allowed to export into a country }\end{array}$ & Estimation method & $\begin{array}{l}\text { Automation and } \\
\text { simplification of } \\
\text { documents compliance } \\
\text { potentially positive impact } \\
\text { on large firms' trade }\end{array}$ \\
\hline $\begin{array}{l}\text { Cheng et } \\
\text { al. (2018) }\end{array}$ & $\begin{array}{l}\text { port-based } \\
\text { evaluation } \\
\text { framework of } \\
\text { trade facilitation } \\
\text { policies }\end{array}$ & $\begin{array}{l}\text { Supply chain dimensions, port, trade } \\
\text { facilitation policies }\end{array}$ & Exploratory & $\begin{array}{l}\text { efficient port infrastructure } \\
\text { has potential impact on } \\
\text { increase in exports }\end{array}$ \\
\hline $\begin{array}{l}\text { Seck } \\
(2016)\end{array}$ & $\begin{array}{l}\text { Trade facilitation } \\
\text { and trade } \\
\text { participation }\end{array}$ & $\begin{array}{l}\text { Customs, import, export, regulation, } \\
\text { finance, equity, share of trading firms } \\
\text { in industry, share of foreign firms in } \\
\text { industry }\end{array}$ & $\begin{array}{l}\text { Benchmark model } \\
\text { (mathematical } \\
\text { equation), pair wise } \\
\text { correlation }\end{array}$ & $\begin{array}{l}\text { Customs procedures and } \\
\text { government regulations } \\
\text { lead to gain better } \\
\text { importing policy that } \\
\text { results in increase in trade } \\
\text { flows }\end{array}$ \\
\hline
\end{tabular}

\section{Conclusion}

Object under study is that of fundamental importance for business communities \& political governments. It is directed concern with economic growth of countries. Problem under investigation is estimation of rank of different countries from within their counterparts. The scope of the problem includes variety of variables viz Logistics performance index, quality of port infrastructure, burden of customs procedures, lead time to export \& imports, documentary compliance cost to export \& import. It is an analysis of 127 countries using the secondary data taken from WDI 2020 using GRA as methodology. It also assemblages the countries on a continuum of a scale of seven items viz exceptionally good, very good, good, fair, poor, very poor and exceptionally poor. Results of GRA show 
that member countries of Organization for Economic Co-operation and Development (OECD) have exceptionally good performance on trade facilitation. Some of member countries of OECD have of very good, some have good performance on trade facilitation. Member countries of Asia Pacific Economic Cooperation (APEC) have fair performance on agenda of trade facilitation. Arabian Countries (AC) have poor trade facilitation. Southern Africa Development Community (SADC) have exceptionally poor trade facilitation. This study contributes towards the body of knowledge by way of ranking of 127 countries with grey relational grades, new additional information by way of grey relational co-efficients and assemblages giving insights and comments at regional bloc levels. It has profound implications for businesses, political governments, researchers and international community because it provides deeper insights and lot of information and facilitates the comparisons. This study also has few limitations. Firstly, cross sectional study in future studies may be longitudinal using time series/panel data. Secondly, this study uses GRA the hierarchical technique there are other hierarchical techniques as well e.g. RIDIT, AHP, TOPSIS, SWARA, VIKOR, ISM future studies may use these methodologies. Thirdly, we have given equal weight to all variables it may be changed on some other basis say on the basis's expert opinion etc. it is considered that different weights specific to the system can be selected for GRA using the multi criteria decision making methods.

\section{References}

Cheng, M. C., Wang, D., \& Wang, J. J. (2018). A port-based evaluation framework of trade facilitation policies: Case of Shenzhen and Hong Kong. Case studies on transport policy, 6(2), 297-307.

Dennis, A. (2010). Global economic crisis and trade: the role of trade facilitation. Applied Economics Letters, 17(18), 1753-1757.

Dennis, A., \& Shepherd, B. (2011). Trade facilitation and export diversification. The World Economy, 34(1), 101-122.

Ertugrul, I., Oztas, T., Ozcil, A., \& Oztas, G. Z. (2016). Grey relational analysis approach in academic performance comparison of university: A case study of Turkish universities. European Scientific Journal, 7881, 128-139.

Feenstra, R. C., \& Ma, H. (2014). Trade facilitation and the extensive margin of exports. The Japanese Economic Review, 65(2), 158-177.

Felipe, J., \& Kumar, U. (2012). The role of trade facilitation in Central Asia: A gravity model. Eastern european economics, 50(4), 5-20.

Fontagné, L., Orefice, G., \& Piermartini, R. (2020). Making small firms happy? The heterogeneous effect of trade facilitation measures. Review of International Economics, 28(3), 565-598.

Gnangnon, S. K. (2017). Impact of trade facilitation reforms on tax revenue. Journal of Economic Studies, 44(5), 765-780.

Hamzaçebi, C., \& Pekkaya, M. (2011). Determining of stock investments with grey relational analysis. Expert Systems with Applications, 38(8), 9186-9195.

Hoekman, B., \& Nicita, A. (2010). Assessing the Doha Round: Market access, transactions costs and aid for trade facilitation. The Journal of International Trade \& Economic Development, 19(1), 65-79.

Indicators, T. F. (2013). The Potential Impact of Trade Facilitation on Developing Countries' Trade. Trade Policy Paper, 144.

Ismail, N. W. (2020). Digital trade facilitation and bilateral trade in selected Asian countries. Studies in Economics and Finance.

Iwanow, T., \& Kirkpatrick, C. (2007). Trade facilitation, regulatory quality and export performance. Journal of International Development: The Journal of the Development Studies Association, 19(6), 735-753.

Jordaan, A. C. (2014). The impact of trade facilitation factors on South Africa's exports to a selection of African countries. Development Southern Africa, 31(4), 591-605.

Julong, D. (1989). Introduction to Grey System Theory. The Journal of Grey System, 1(1), 1-24.

Kuo, Y., Yang, T., \& Huang, G. W. (2008). The use of grey relational analysis in solving multiple attribute decision-making problems. Computers \& industrial engineering, 55(1), 80-93.

Lesser, C., \& Moisé-Leeman, E. (2009). Informal cross-border trade and trade facilitation reform in 
Sub-Saharan Africa.

Lorz, O. (2020). Investment in trade facilitating infrastructure: A political-economy analysis. European Journal of Political Economy, 65, 101928.

Manzoor, R., Maken, A. M., Syed, S. A., \& Ahmed, V. (2019). Trading with India: Some current impediments for Pakistan. Journal of International Trade Law and Policy.

Marti, L., Puertas, R., \& García, L. (2014). Relevance of trade facilitation in emerging countries' exports. The Journal of International Trade \& Economic Development, 23(2), 202-222.

Martinez-Zarzoso, I., \& Márquez-Ramos, L. (2008). The effect of trade facilitation on sectoral trade. The BE Journal of Economic Analysis \& Policy, 8(1).

Martínez- Zarzoso, I., \& Rudolf, R. (2020). The trade facilitation impact of the Chinese diaspora. The World Economy.

Messerlin, P. A., \& Zarrouk, J. (2000). Trade Facilitation: Technical regulations and customs procedures. World Economy, 23(4), 577-593.

Nguyen Viet, C. (2015). The impact of trade facilitation on poverty and inequality: Evidence from lowand middle-income countries. The Journal of International Trade \& Economic Development, 24(3), 315-340.

Park, H. G., \& Park, Y. (2018). Examining the perceived impact of financial investment in AEO certification on performance through trade facilitation and public and private partnership. The International Journal of Logistics Management, 29, 46-63.

Qazi, T. F.,Basit, A.,Asghar, W.\& Niazi, A. A. K.(2020).Composite Appraisal of Women Development in Selected Thirty-six Countries with Special Focus on Pakistan: Applying Grey Incidence Analysis Model. Journal of Businessand Social Review in Emerging Economies, 6(4), 1227-1236

Sakyi, D., Bonuedi, I., \& Opoku, E. E. O. (2018). Trade facilitation and social welfare in Africa. Journal of African Trade, 5(1-2), 35-53.

Seck, A. (2016). Trade facilitation and trade participation: Are sub-Saharan African firms different? Journal of African trade, 3(1-2), 23-39.

Shepherd, B. (2016). Did APEC's Trade Facilitation Action Plans deliver the goods?. Journal of Asian Economics, 43, 1-11.

Shepherd, B. (2016). Infrastructure, trade facilitation, and network connectivity in Sub-Saharan Africa. Journal of African trade, 3(1-2), 1-22.

Tang, R. (2020). Does trade facilitation promote the efficiency of inbound tourism?-The empirical test based on Japan. International Journal of Tourism Research.

Tayyar, N., Akcanlı, F., Genç, E., \& Erem, I. (2014). Evaluating the financial performance of companies operating in the field of informatics and technology registered in BIST by analytical hierarchy process (AHP) and gray relational analysis (TIA) method. Accounting and Finance Journal, 61, $19-40$.

Tung, R. L., \& Chung, H. F. (2010). Diaspora and trade facilitation: The case of ethnic Chinese in Australia. Asia Pacific Journal of Management, 27(3), 371-392.

Ural Marchand, B. (2019). Inequality and Trade Policy: The Pro- Poor Bias of Contemporary Trade Restrictions. Review of Income and Wealth, 65, S123-S152.

Valensisi, G., Lisinge, R., \& Karingi, S. (2016). The trade facilitation agreement and Africa's regional integration. Canadian Journal of Development Studies/Revue canadienne d'études du développement, 37(2), 239-259.

Wan, J., Baylis, K., \& Mulder, P. (2015). Trade-facilitated technology spillovers in energy productivity convergence processes across EU countries. Energy Economics, 48, 253-264.

Wei, G. (2011). Grey Relational Analysis Model for Dynamic Hybrid Multiple Attribute Decision Making. Knowledge-Based Systems, 24(5), 672- 679.

Wilson, J. S., Mann, C. L., \& Otsuki, T. (2005). Assessing the benefits of trade facilitation: A global perspective. World Economy, 28(6), 841-871.

World Development Indicators. (2020). Retrieved April 11, 2020, from http://wdi.worldbank.org/tables

Wu, H. H. (2002). A comparative study of using grey relational analysis in multiple attribute decision making problems. Quality Engineering, 15(2), 209-217. 
Zaki, C. (2013). Trade facilitation and corruption: a CGE model of Egypt. The Journal of North African Studies, 18(1), 70-111. 